\title{
policystatement
}

\section{Duty to re-contact}

\section{INTRODUCTION}

Rapid and continued advances in human genetics have resulted in new opportunities for the early detection of disease, presymptomatic diagnosis, and the possibility for new interventions and therapies. The vast majority of physicians would agree that optimal medical care calls for informing patients of these new developments. The difficult issue is determining which physician or other health professional should be responsible for informing the patient of advances which become available after the last scheduled consultation.

The current climate of health care has vested primary care physicians with responsibility for the medical management of the whole family. Therefore, families can now expect directions and directives about their health from their own primary care physicians. Specialist consultations arranged by primary physicians serve to enhance care, but the patient ultimately returns to his/her primary physician for continued management. Cost considerations have driven the evolution of this approach, as well as the increased use of nonphysician medical personnel for patient education and management of care. Ultimately, the responsibility to adequately inform and care for patients falls to the physician. The question is which physician bears the burden of continuing education of patients?

\section{ISSUES}

- Whose responsibility is it to re-contact the patient-the primary care physician or the consulting geneticist?

- When should a re-contact or referral for an updated consultation occur?

- Can the patient be the responsible party?

- Whose responsibility is it to amend the genetic counseling?

- Is there a liability for not contacting the family?

\section{DISCUSSION}

Clearly, it is unarguable that during a consultation the medical geneticist is expected to inform the patient of all the relevant dimensions of diagnosis, care, and possible therapy. Difficulty is encountered when advances occur months or years after the original consultation. Except in those instances in which continuing care, even on an annual basis, is rendered by a medical geneticist, common sense dictates that the physician providing continual care (usually the primary care physician) becomes the responsible party for informing the family of the need to re-contact the geneticist. The primary care doctor is expected to know the family and ask if new developments in the family history have occurred and to be alert to new potential opportunities that could affect the health and future of his/her patients. Under ordinary circumstances, a medical geneticist does not maintain ongoing contact with patients and would be hard-pressed to locate patients years later to advise them of new advances. The impracticability of this endeavor is illustrated by the fact that some 40 million people change their addresses each year. Given the continuous movement of families, it is more appropriate for families to establish their "genetic connections" with the primary care doctor with whom they register. Medical geneticists may also relocate during their careers and cannot be expected to continue responsibilities from afar. While successors assume overall responsibilities, it is still more rational for patients and their own doctors to be vested with the duty of recontacting the medical geneticists for updates as appropriate.

In terms of when and if a re-contact should occur, there is no set specified time requirement. However, in the event of changes in the life cycle such as marriage, or childbearing, or when questions are posed by the patient, any updated information should be provided. If a patient is seen by their primary care physician on a yearly basis, updates from the initial consultation can be addressed. The primary care physician can elicit if there are new advances by a telephone conversation between themselves and the genetics unit and, if necessary, a new referral for a genetics consultation should be provided to the patient.

The patient(s) can have an integral role in this process. The geneticist, as part of his/her responsibility, should provide a written summary of the consultation to the physician and patient as appropriate. ${ }^{*}$ The patient should be adequately counseled, and the recommendation should be clearly stated in the letter that should any changes occur (i.e., a pregnancy) they should alert their primary care physician and/or contact the genetics unit so that appropriate care can be provided. This approach reinforces the principle that patient and provider are partners in assuming optimal outcome.

There are now many examples in the face of remarkable advances in human genetics in which genetic counseling given just a few years previously is now known to be incorrect. For example, a disorder thought to be multifactorial is now recognized as monogenic, or an idiopathic disorder is now recognized as a microdeletion syndrome. In such instances, although medical geneticists are likely to be the first to recognize the implications for the patients they had previously studied, timely questions by the family to the primary care provider would lead to an inquiry to the medical geneticist and/or the laboratory.

\section{SUMMARY}

After an initial genetics consultation, the referring physician and, as appropriate, the patient and the designated primary care

\footnotetext{
* Although the Board of the American College of Medical Genetics has authorized the publication of this Statement by the Social, Ethical and Legal Issues Committee, there was disagreement among the Board nembers as to whether there is responsibility in all cases to provide a written summary. Part of the Statement therefore should not be interpreted as a rule but only as a recommendation.
} 
provider should receive a written summary of the consultation that includes the recommendation to contact the genetics unit for new advances. ${ }^{*}$ It is the medical geneticist's responsibility to provide clinical updates to those patients to whom they provide an on-going service. However, since this represents the smaller percentage of the caseload, it should be incumbent upon the primary care physician to alert his/her patient to the need for a recontact as necessary. The patient should also be included in the process by being adequately counseled to contact the primary care physician or genetics unit as relevant changes in their lives occur.
Social Ethical and Legal Issues Committee
American College of Medical Genetics
Kurt Hirschhorn, MD, Chair
Lynn D. Fleisher, PhD, JD
Lynn Godmilow, MSW
R. Rodney Howell, MD
Robert R. Lebel, MD
E. R. B. McCabe, MD
Matthew J. McGinniss, PhD
Aubrey Milunsky, MD
Mary Z. Pelias, PhD, JD
Reed E. Pyeritz, MD, PhD
Eva Sujansky, MD
Barry H. Thompson, MD
Randi-Ellen Zinberg, MS

This guideline is designed primarily as an educational resource for medical geneticists and other health care providers to help them provide quality medical genetic services. Adherence to this guideline does not necessarily assure a successful medical outcome. This guideline should not be considered inclusive of all proper procedures and tests or exclusive of other procedures and tests that are reasonably directed to obtaining the same results. In determining the propriety of any specific procedure or test, the geneticists should apply his or her own professional judgment to the specific clinical circumstances presented by the individual patient or specimen. It may be prudent, however, to document in the patient's record the rationale for any significant deviation from this guideline. 